\title{
Giant unruptured sinus valsalva aneurysm: an unusual cause of typical angina
}

\section{Felix Schönrath'*, Hatem Alkadhi², Ulf Landmesser ${ }^{3}$, Volkmar Falk', and André Plass'}

${ }^{1}$ Clinic for Cardiovascular Surgery, University Hospital of Zurich, Zurich, Switzerland; ${ }^{2}$ Clinic for Diagnostic and Interventional Radiology, University Hospital of Zurich, Zurich, Switzerland; and ${ }^{3}$ Clinic for Cardiology, University Hospital of Zurich, Zurich, Switzerland

*Corresponding author. Tel: +41 44 2553298, Fax: +41 44 2554446. Email: felix.schoenrath@usz.ch

This paper was guest edited by Brahmajee Karthik Nallamothu, University of Michigan, Ann Arbor, MI, USA

A 67-year-old man presented with dyspnoea and angina symptoms in a peripheral hospital. A CT scan of the chest was performed because of suspicion of pulmonary embolism. After application of the contrast agent, an anaphylactic shock occurred. After successful reanimation, a transoesophageal echocardiography displayed a large sinus Valsalva aneurysm (diameter $75 \mathrm{~mm}$ ). Thereafter, the patient was referred to our hospital.

Magnetic resonance imaging demonstrated a close anatomical relation between the aneurysm and the left coronary artery (Panel $B$ : arrow-sinus Valsalva aneurysm, Panel C: arrow-left main artery, asterisk-sinus Valsalva aneurysm). A coronary angiogram was performed and revealed occlusion of the left main artery caused by the large unruptured aneurysm. The left coronary system filled partly retrograde (Panels $D$ and $E$, Supplementary material online, Videos S2 and S3). Echocardiography during open heart surgery showed the aneurysm to be filled with a mobile thrombus (Panel A, Supplementary material online, Video S1). The intra-operative assessment of the aneurysm showed the origin inferior to the left main coronary artery continuing laterally just posterior to the pulmonary artery (Panel F, black arrow: aneurysm, white arrow and Panel Gthrombus). Because the aneurysm adhered to the left coronary ostium, coronary bypass grafts to the left anterior descending artery and circumflex artery were performed
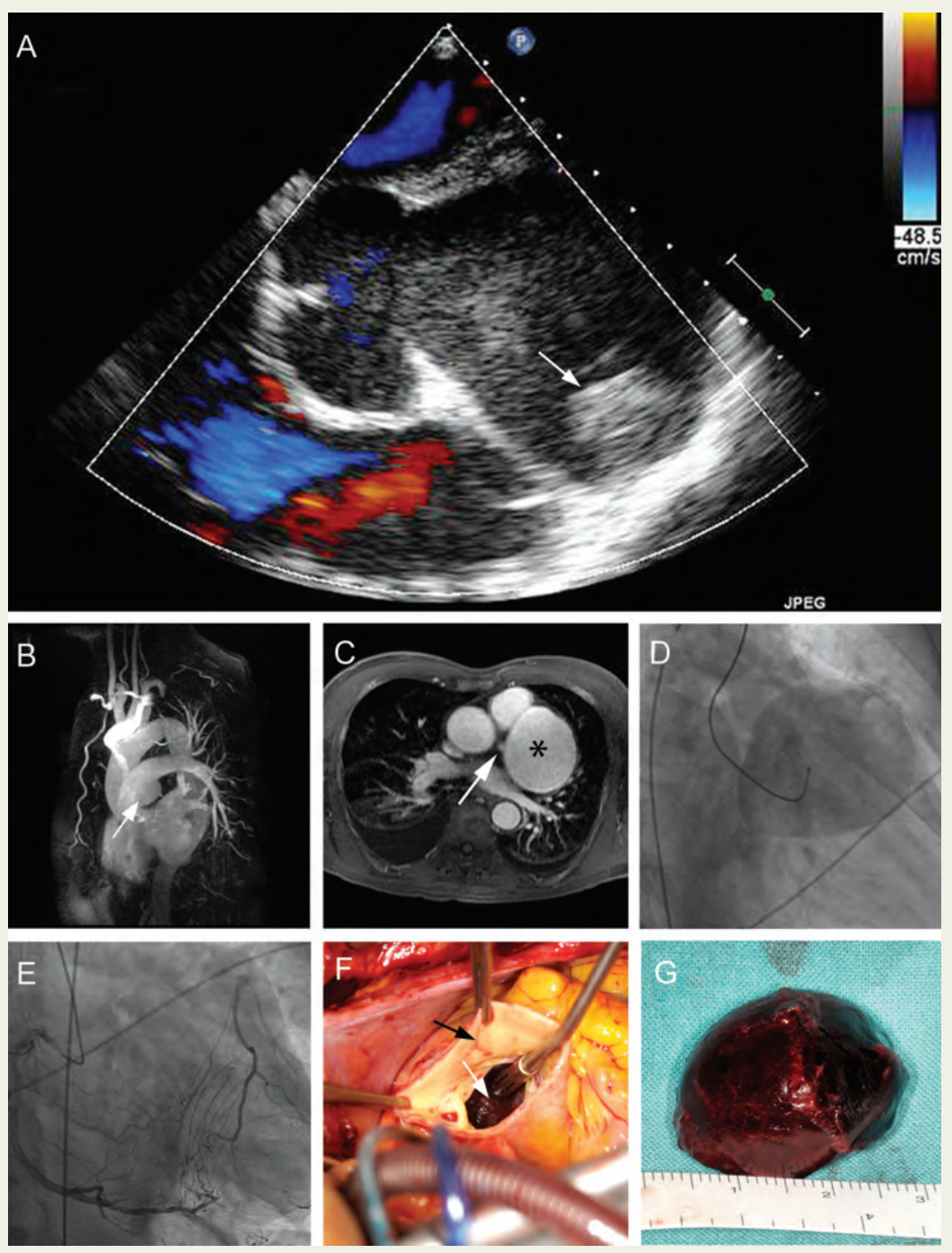

using the left (LIMA) and right (as T-Graft from LIMA) mammary arteries. The aneurysm was resected and the aortic root was replaced with a biological composite graft. The patient had a post-operative course without complication and a fast recovery.

Supplementary material is available at European Heart Journal online. 